\title{
Dual Pathologies of Parathyroid Adenoma and Papillary Thyroid Cancer on Fluorocholine and Fluorodeoxyglucose PET/CT
}

\author{
N. T. K. Thanseer ${ }^{1} \cdot$ Sanjay Kumar Bhadada $^{2} \cdot$ Ashwani Sood $^{1}$ (D) \\ Ashwin Singh Parihar ${ }^{1} \cdot$ Divya Dahiya $^{3}$ - Priyanka Singh ${ }^{2} \cdot$ Rajender Kumar Basher $^{1}$. \\ Ashim Das ${ }^{4}$ - Bhagwant R. Mittal ${ }^{1}$
}

Received: 28 April 2017 / Revised: 11 July 2017 / Accepted: 13 July 2017 / Published online: 25 July 2017

(C) Korean Society of Nuclear Medicine 2017

\begin{abstract}
F}$-Fluorocholine (FCH) PET/CT is evolving as a functional imaging modality for the preoperative imaging of abnormal parathyroid tissue(s) helping to localize eutopic and ectopic parathyroid tissue and limit the extent of surgery. FCH PET/CT may show incidental uptake in various thyroid lesions necessitating further evaluation, whereas the role of ${ }^{18}$ F-fluorodeoxyglucose (FDG) PET/CT in the detection of incidental thyroid nodules is well documented. The case of a middle-aged woman with dual pathology of parathyroid adenoma and papillary thyroid cancer detected on FCH and FDG $\mathrm{PET} / \mathrm{CT}$ is presented.
\end{abstract}

Keywords Parathyroid adenoma $\cdot$ Papillary thyroid carcinoma $\cdot{ }^{18}$ F-Fluorocholine $\cdot{ }^{18}$ F-Fluorodeoxyglucose . $\mathrm{PET} / \mathrm{CT}$

The original version of this article was revised to correct the name of the fifth author.

Ashwani Sood

sood99@yahoo.com

1 Department of Nuclear Medicine, PGIMER, Sector-12, Chandigarh 160012, India

2 Department of Endocrinology, PGIMER, Chandigarh 160012, India

3 Department of General Surgery, PGIMER, Chandigarh 160012, India

4 Department of Histopathology, PGIMER, Chandigarh 160012, India

\section{Introduction}

${ }^{18} \mathrm{~F}$-Fluorocholine (FCH) PET/CT is a promising modality for the detection of abnormal parathyroid tissue(s) [1]. FCH PET/ CT may show variable tracer uptake in different incidentally detected benign and malignant lesions in the thyroid gland. FCH taken up by the thyroid gland during parathyroid imaging requires further evaluation as the uptake may be pathological [2-6]. While the incidental finding of focal FDG uptake in the thyroid gland on whole-body FDG PET/CT done for other conditions might indicate a malignant lesion requiring further work-up, the absence of FDG uptake in a large thyroid nodule nearly excludes malignancy [7-9]. We present the case of middle-aged woman in whom functional imaging studies helped detect the dual pathology of parathyroid adenoma and papillary thyroid carcinoma (PTC).

\section{Case Report}

A 56-year-old woman presented with epigastric pain and vomiting (occasionally bilious) for 5 days with similar episodes in the past 2 years. Her biochemistry work-up revealed elevated calcium $(12.3 \mathrm{mg} / \mathrm{dL}$, normal range $8.8-10.2 \mathrm{mg} /$ $\mathrm{dL})$, alkaline phosphatase (212.94 U/L, normal range 42$128 \mathrm{U} / \mathrm{L}$ ) and intact parathyroid hormone (PTH) level (167 pg/mL, normal range $15-65 \mathrm{pg} / \mathrm{mL}$ ), and a low phosphate level $(2.47 \mathrm{mg} / \mathrm{dL}$, normal range $2.7-4.5 \mathrm{mg} / \mathrm{dL})$. She was managed symptomatically with a provisional diagnosis of acute on chronic pancreatitis secondary to the raised PTH 
Fig. 1 a, b ${ }^{99 \mathrm{~m}} \mathrm{Tc}-$ Sestamibi dual-phase planar images show a focal tracer-avid lesion (arrows) below the left thyroid lobe on both the early (a) and delayed (b) images. c, d SPECT/CT images of the neck and mediastinum: transaxial CT (c) and fused (d) images show a small soft-tissue lesion with tracer avidity inferior to the left thyroid lobe
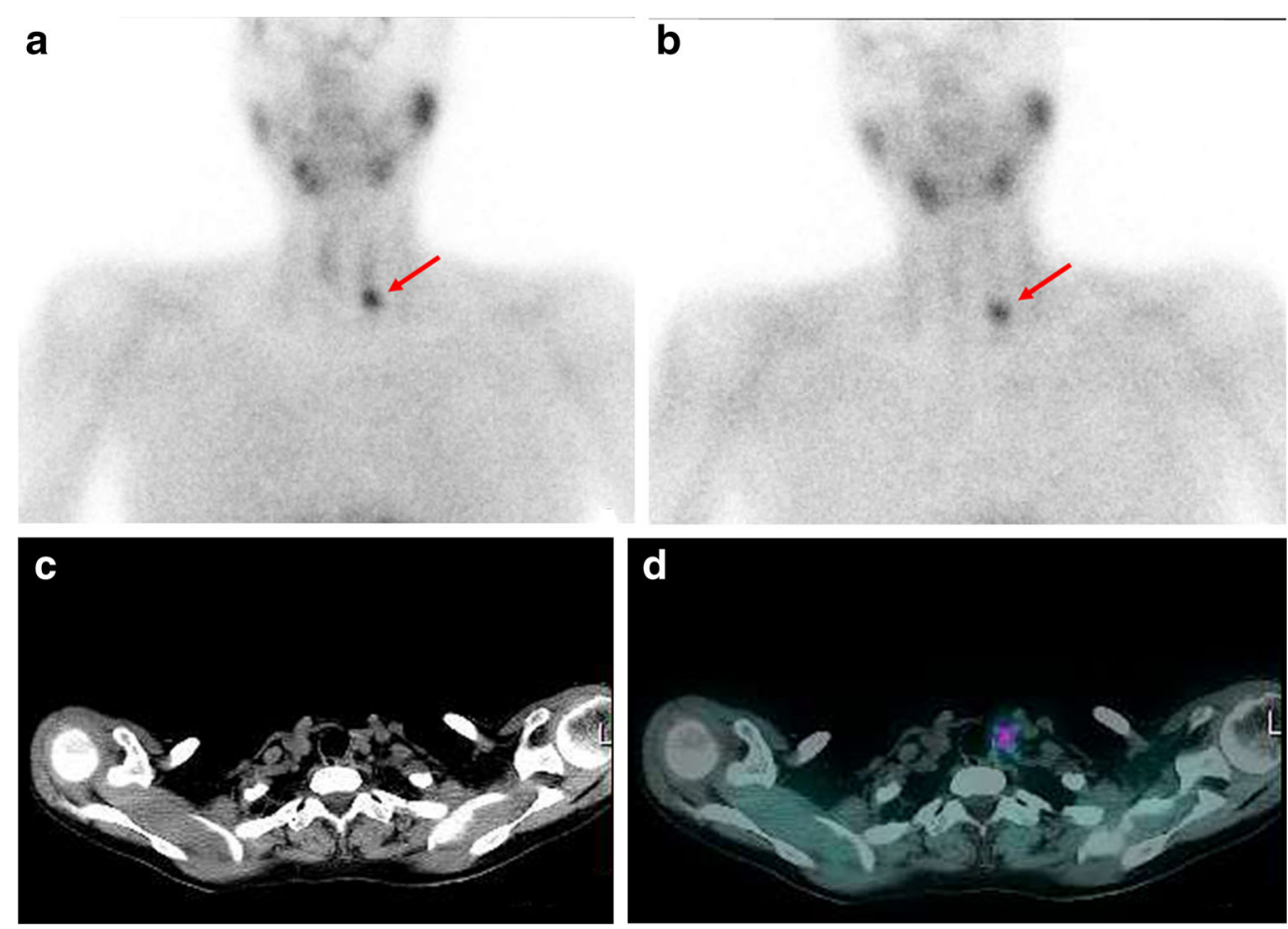

level. Neck ultrasonography showed a hypoechoic soft-tissue lesion inferior to the left thyroid lobe with multiple small hypoechoic lesions in the right upper thyroid lobe. ${ }^{99 \mathrm{~m}} \mathrm{Tc}-$ Sestamibi (MIBI) planar imaging of the neck and mediastinum (Fig. 1a, b) showed a focal tracer-avid lesion below the left thyroid lobe on both early and delayed images. Transaxial CT and SPECT fused images showed a soft-tissue lesion with tracer avidity inferior to the left thyroid lobe (Fig. 1c, d) suggestive of left inferior parathyroid adenoma.

An FCH PET/CT scan performed after intravenous injection of $185 \mathrm{MBq}$ of radiopharmaceutical as part of an ongoing project at our institution showed a choline-avid focus (SUVmax about 16) in the left lower neck and a faint choline-avid focus in the right upper neck on the maximum intensity projection (MIP) image (Fig. 2a) of the neck and mediastinum. Transaxial CT and fused images showed a faint choline-avid hypodense lesion in the right thyroid lobe and an intense choline-avid soft-tissue lesion inferior to the left thyroid lobe (Fig. 2c-g). The MIP image (Fig. 2b) of the FDG PET/CT scan of the neck and mediastinum showed an FDGavid focus (SUVmax about 16) in the right upper neck. Transaxial CT and fused images showed an intense FDGavid hypodense lesion in the right thyroid lobe and a non-
FDG-avid soft-tissue lesion inferior to the left thyroid lobe (Fig. 2c-h).

FDG PET/CT performed for thyroid nodule characterization and staging of the neck showed an intense FDG-avid hypodense lesion in the right thyroid lobe and no FDG uptake in the left parathyroid adenoma, while FCH PET/CT revealed mild $\mathrm{FCH}$ avidity in the lesion in the right upper lobe of the thyroid gland and an intense FCH-avid left inferior parathyroid adenoma. Ultrasound-guided fine-needle aspiration cytology of the right upper lobe thyroid lesion (intense FDG and mild FCH avidity) revealed the presence of PTC. The patient underwent right hemithyroidectomy for PTC and excision of the left inferior parathyroid adenoma.

Histopathology of the surgical specimens confirmed the preoperative findings of PTC and parathyroid adenoma with histological sections showing the papillary configuration of the PTC (Fig. 3a) and the nuclear features of the PTC in the form of optical clearing, overcrowding and grooving (Fig. 3b). Figure $3 \mathrm{c}$ shows a histological section of the normal parathyroid gland, and Fig. $3 \mathrm{~d}$ shows the parathyroid adenoma with the inset showing the adenoma and normal parathyroid at the periphery of the adenoma. The serum PTH level $(32 \mathrm{pg} / \mathrm{ml})$ and calcium level $(8.8 \mathrm{ng} / \mathrm{ml})$ became normal on the third 
Fig. 2 a The ${ }^{18}$ F-FCH PET/CT MIP image shows an intense tracer-avid focus (thick arrow) in the left lower neck and a faint tracer-avid focus (thin arrow) in the right upper neck. $\mathbf{c}, \mathbf{d}, \mathbf{f}, \mathbf{g}$ The transaxial $\mathrm{CT}$ and fused images show a right thyroid lobe hypodense lesion with faint tracer uptake and a soft-tissue lesion inferior to the left thyroid lobe with intense tracer uptake. $\mathbf{b}^{18} \mathrm{~F}$ FDG PET/CT MIP image shows a tracer-avid focus (thin arrow) in the right upper neck. $\mathbf{c}, \mathbf{e}, \mathbf{f}, \mathbf{h}$ Transaxial CT and fused images show a right thyroid lobe hypodense lesion with intense tracer uptake and a small softtissue lesion with no tracer uptake inferior to the left thyroid lobe
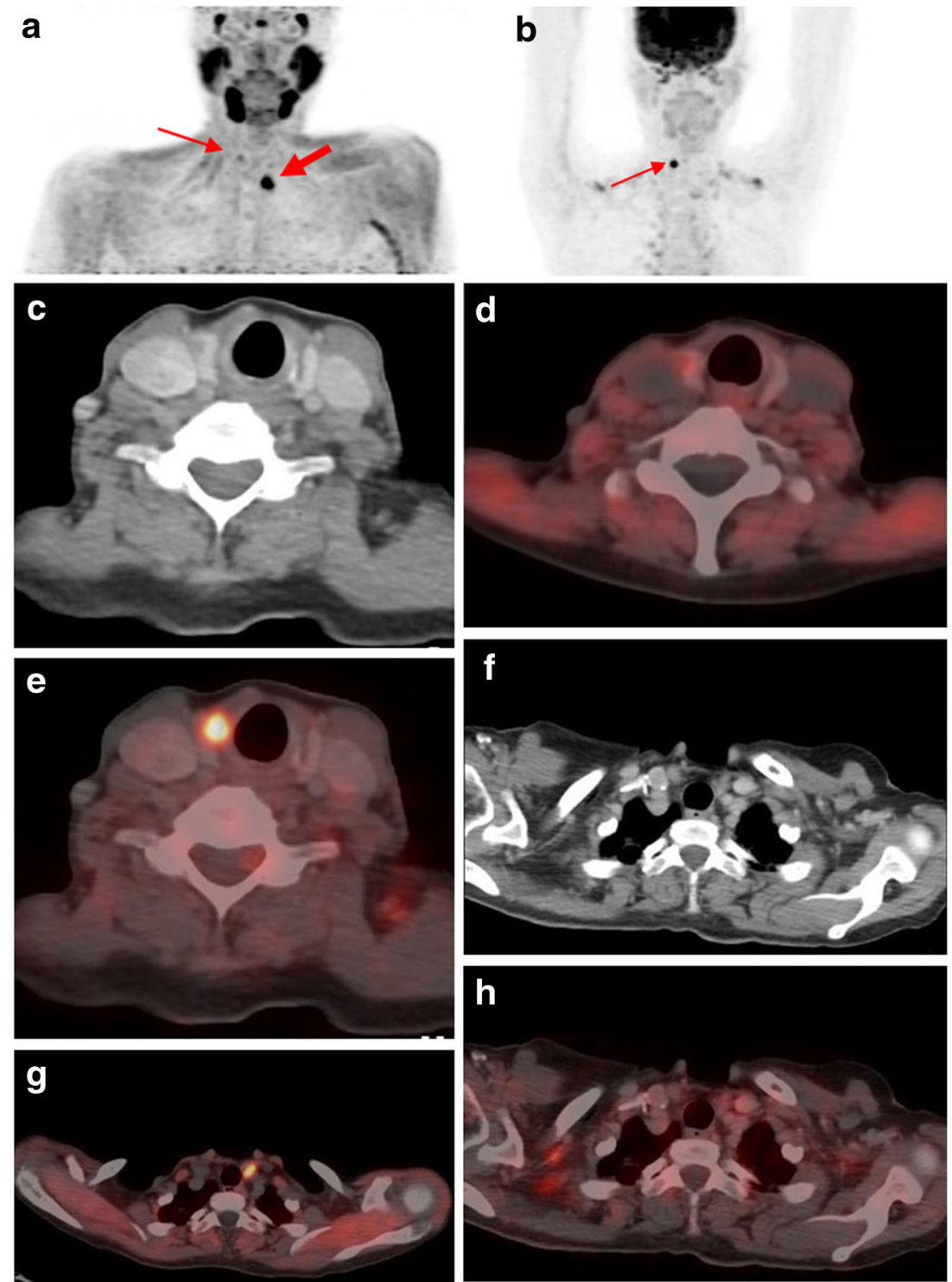

postoperative day. At the time of this report, the patient was asymptomatic with a normal biochemical profile.

\section{Discussion}

Choline, a precursor in phosphatidylcholine biosynthesis in cellular membranes with upregulation of choline kinase in tumor, as well as its use in the evaluation of prostate cancer, shows uptake in various benign and neoplastic lesions [10]. FCH PET/CT is emerging as a novel imaging modality for the detection of parathyroid lesions [1]. However, additional pathologies including thyroid lymphoma, differentiated thyroid carcinoma, lymph node metastases from tall-cell thyroid cancer, Hürthle cell adenoma and oncocytic adenoma of the thyroid show variable FCH uptake and have been detected on FCH imaging [2-6]. FDG, a glucose metabolic marker, has demonstrated focally increased uptake in incidentally detected thyroid lesions with more than one-third of them being malignant, but performs poorly in the localization of the parathyroid gland $[7,8]$. A negative FDG PET/CT study in 

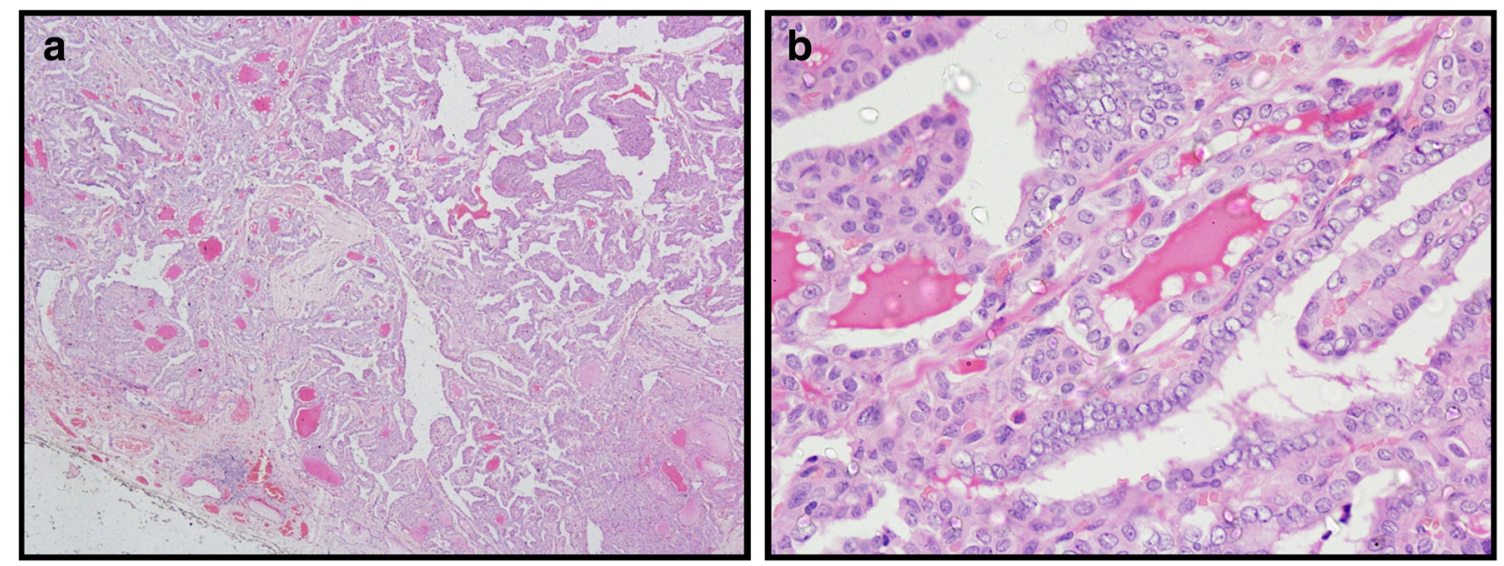

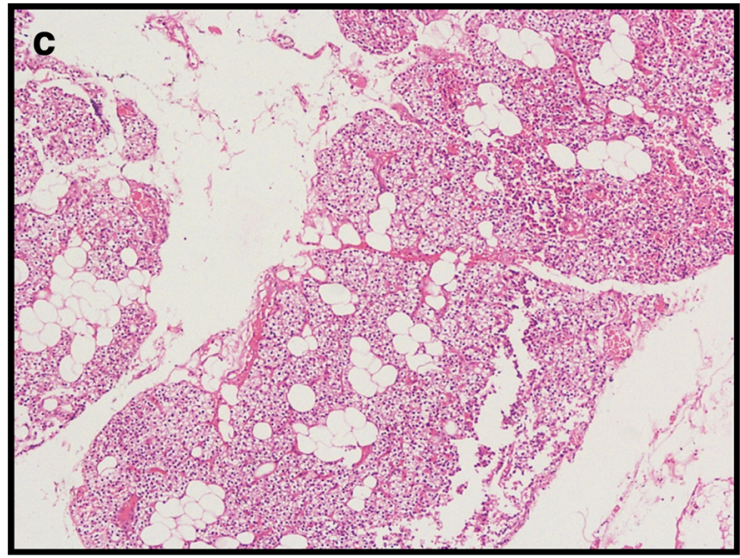

Fig. 3 Histopathological examination of the surgical specimens. a, b Photomicrographs of H\&E-stained sections show (a) the papillary configuration of the papillary thyroid carcinoma $(\times 40)$ and $(b)$ the nuclear features of the papillary thyroid carcinoma in the form of

the evaluation of suspicious thyroid nodules shows high negative predictive value for malignancy particularly in larger lesions [9].

The subject of the case presented here showed an FCH-avid, MIBI-avid and non-FDG-avid left inferior parathyroid adenoma. The incidentally detected right thyroid nodule showed intense FDG avidity and mild FCH avidity and the postoperative histopathology showed PTC. The findings reported here are different from those of a study of four newly diagnosed patients with recurrent thyroid cancer in whom the primary disease, and locoregional and lung metastases showed choline avidity (SUVmax $7.9 \pm 4.9$ ) and absent or mild FDG uptake. Similarly aggressive lymph node metastases from a thyroid malignancy have shown choline avidity without FDG avidity in a case report $[4,6]$. These findings indicate that benign as well as malignant thyroid lesions may show differential FDG and FCH

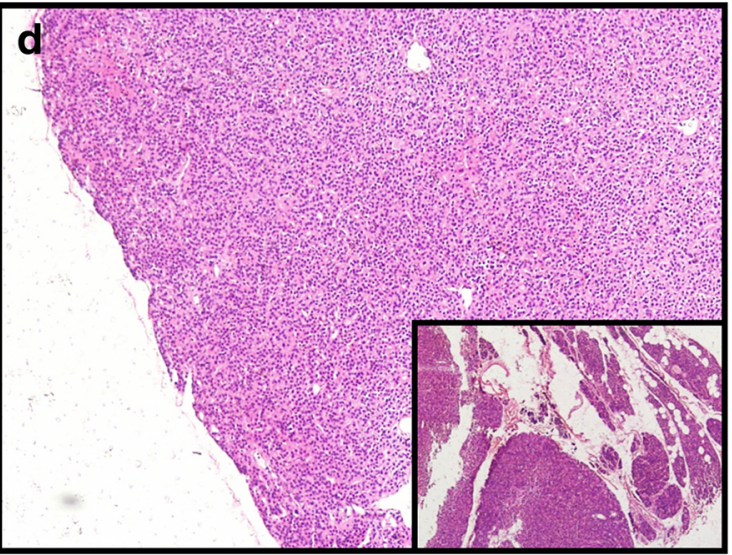

optical clearing, overcrowding and grooving $(\times 400)$. c, d Photomicrographs of H\&E-stained sections show (c) normal parathyroid gland $(\times 100)$, and $(\mathbf{d})$ normal parathyroid at the periphery of the adenoma $(\times 40)$ and inset the adenoma

uptake, making the complementary use of both imaging modalities worthwhile in the situation of a patient with dual pathology.

This case report emphasizes the significance of the dual pattern of high FDG and low FCH uptake in an incidentally detected thyroid malignancy with absent FDG and high FCH uptake in parathyroid adenoma in a patient requiring appropriate evaluation.

\section{Compliance with Ethical Standards}

\section{Conflicts of Interest None.}

Ethical Approval All procedures performed in studies involving human participants were in accordance with the ethical standards of the institutional and/or national research committee and with the principles 
of the 1964 Declaration of Helsinki and its later amendments or comparable ethical standards.

Informed Consent The institutional review board of our institution approved this retrospective study, and the requirement to obtain informed consent was waived.

\section{References}

1. Lezaic L, Rep S, Sever MJ, Kocjan T, Hocevar M, Fettich J. 18FFluorocholine PET/CT for localization of hyperfunctioning parathyroid tissue in primary hyperparathyroidism: a pilot study. Eur J Nucl Med Mol Imaging. 2014;41:2083-9.

2. Eccles A, Challapalli A, Khan S, Barwick T, Mangar S. Thyroid lymphoma incidentally detected by 18 F-fluorocholine (FCH) PET/ CT. Clin Nucl Med. 2013;38:755-7.

3. Aziz AL, Courbon F, Dierickx LO, Pascal P, Zerdoud S. Oncocytic adenoma of thyroid incidentally detected by $18 \mathrm{~F}$-fluorocholine PET/CT. J Nucl Med Technol. 2015;43:133-4.

4. Wu HB, Wang QS, Wang MF, Li HS. Utility of 11C-choline imaging as a supplement to F-18 FDG PET imaging for detection of thyroid carcinoma. Clin Nucl Med. 2011;36:91-5.
5. Paone G, Treglia G, Bongiovanni M, Ruberto T, Ceriani L, Giovanella L. Incidental detection of Hürthle cell adenoma by $18 \mathrm{~F}$-choline PET/CT scan in a patient with prostate cancer. Rev Esp Med Nucl Imagen Mol. 2013;32:340-1.

6. Piccardo A, Massollo M, Bandelloni R, Arlandini A, Foppiani L. Lymph node metastasis from tall-cell thyroid cancer negative on 18F-FDG PET/CT and detected by $18 \mathrm{~F}$-choline PET/CT. Clin Nucl Med. 2015;40:e417-9.

7. Soelberg KK, Bonnema SJ, Brix TH, Hegedüs L. Risk of malignancy in thyroid incidentalomas detected by $18 \mathrm{~F}$ fluorodeoxyglucose positron emission tomography; a systematic review. Thyroid. 2012;22:918-25.

8. Hindie E, Ugur O, Fuster D, O'Doherty M, Grassetto G, Urena P, et al. 2009 EANM parathyroid guidelines. Eur J Nucl Med Mol Imaging. 2009;36:1201-16.

9. Vriens D, de Wilt JH, van der Wilt GJ, Netea-Maier RT, Oyen WJ, de Geus-Oei LF. The role of [18F]-2-fluoro-2-deoxy-d-glucosepositron emission tomography in thyroid nodules with indeterminate fine-needle aspiration biopsy: systematic review and metaanalysis of the literature. Cancer. 2011;117:4582-94.

10. Savelli G, Basile P, Muni A, Bna C, Pizzocaro C, Pagani R. Focal thyroid uptake during $18 \mathrm{~F}$-choline PET/CT: a case report. Case Rep Clin Med. 2015;4:345-8. 